\title{
III-V-on-Silicon Filtered Feedback Discretely Tunable Laser with Nanosecond Switching Times
}

\author{
Sören Dhoore ${ }^{1,2}$, Abdul Rahim ${ }^{1,2}$, Gunther Roelkens ${ }^{1,2}$, and Geert Morthier ${ }^{1,2}$ \\ ${ }_{1}^{1}$ Photonics Research Group, INTEC, Ghent University-IMEC, B-9052, Ghent, Belgium \\ ${ }^{2}$ Center for Nano-and Biophotonics (NB-Photonics), Ghent University, B-9052, Ghent, Belgium \\ Soren.Dhoore@UGent.be
}

\begin{abstract}
We demonstrate nanosecond fast wavelength switching with a heterogeneously integrated III-V-on-silicon four-channel filtered feedback tunable laser. High-speed direct modulation at $12.5 \mathrm{Gbit} / \mathrm{s}$ of each wavelength channel is achieved. ( 2018 The Author(s)
\end{abstract}

OCIS codes: (140.3600) Lasers, tunable, (230.7408) Wavelength filtering devices, (250.5300) Photonic integrated circuits.

\section{Introduction}

Datacenter networks based on optical packet or burst switching are expected to become a reality in the near future [1]. Envisioned implementations of such network architectures are typically based on passive wavelength routing devices and fast tunable lasers with tuning speeds on the order of several nanoseconds. Recently, we have experimentally demonstrated over $12 \mathrm{~nm}$ continuous wavelength tunability through current injection into the passive tuning layer of a tunable twin-guide (TTG) membrane of a novel III-V-on-silicon distributed Bragg reflector (DBR) laser structure [2]. Tuning was, however, mainly thermal (and thus slow) because of the limited confinement factor of the optical mode in the tuning layer and because of the device's large thermal resistance. In this paper, we demonstrate for the first time nanosecond fast wavelength switching with an AWG-based III-V-on-silicon laser structure that uses the filtered feedback principle to achieve single-mode lasing operation and tunability. The concept was first introduced and experimentally demonstrated by B. Docter et al. [3], where discrete wavelength tuning was achieved with a pure InP/InGaAsP-based laser. The implementation on silicon, however, allows to benefit from the superior passive functionality of silicon, leading to improved static and dynamic lasing characteristics, as supported by our experimental results.
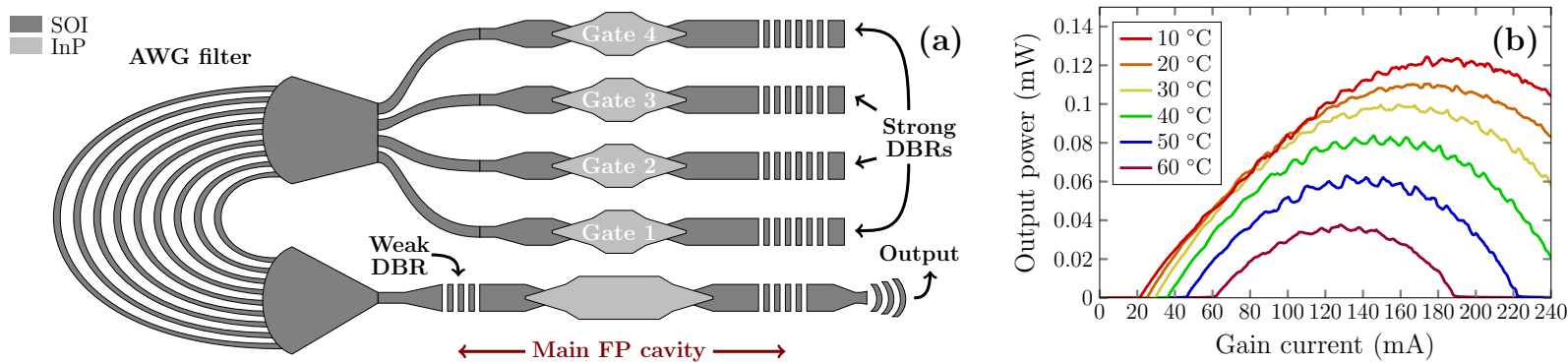

Fig. 1. (a) Schematic view of the demonstrated laser structure. (b) LI-curves at different stage temperatures with unbiased gates (fiber-coupled output power versus gain current).

\section{Laser Design and Fabrication}

The laser structure is schematically shown in Fig. 1(a). The laser consists of a main Fabry-Pérot (FP) cavity with two partially reflective broadband silicon-on-insulator (SOI) DBR mirrors. The light output at one side of the FP cavity is coupled to an SOI arrayed waveguide grating (AWG) filter that splits the light in four different waveguide branches. In each branch a III-V-on-silicon semiconductor optical amplifier (SOA) is implemented that can function as optical gate. By making use of a highly reflective SOI DBR mirror at the end of each waveguide branch, light can be fed back to 
the laser, enabling the laser to operate in single-mode regime. The AWG has a free spectral range (FSR) of $20 \mathrm{~nm}$ with a channel spacing of $4 \mathrm{~nm}$ and an extinction ratio of more than $10 \mathrm{~dB}$. The FP main cavity and SOA gates (including III-V-to-silicon couplers) have a length of $600 \mu \mathrm{m}$ and $400 \mu \mathrm{m}$ respectively. The SOI structures have a $400 \mathrm{~nm}$ thick silicon device layer with an etch depth of $180 \mathrm{~nm}$ and were fabricated in-house by means of electron-beam lithography (EBL) and reactive ion etching (RIE). III-V-on-silicon integration is based on adhesive DVS-BCB bonding. Details of the bonding procedure and III-V post processing can for instance be found in [4].

\section{Characterization Results}

Fig. 1(b) shows the light-current (LI) characteristics of the laser device. At room temperature the laser threshold is $20 \mathrm{~mA}$, with a maximum fiber-coupled output power of $0.1 \mathrm{~mW}$. When the SOA gates are biased above transparancy (gate current $\sim 50 \mathrm{~mA}$ ) single-mode lasing operation can be achieved with a SMSR larger than $35 \mathrm{~dB}$ (see Figs. 2(a)(c)). Dynamic characterization results are shown in Figs. 2(d)-(f). As proof-of-principle wavelength tuning is done by switching between Gate 3 and Gate 4 . A switching speed of several nanoseconds is obtained. Finally, large-signal measurements indicate back-to-back operation at $12.5 \mathrm{Gbit} / \mathrm{s}$ non-return-to-zero on-off keying (NRZ-OOK) direct modulation for all four wavelength channels with a BER $\sim 10^{-5}$, which is well below the $7 \%$ hard-decision forward error correction (HD-FEC) limit.
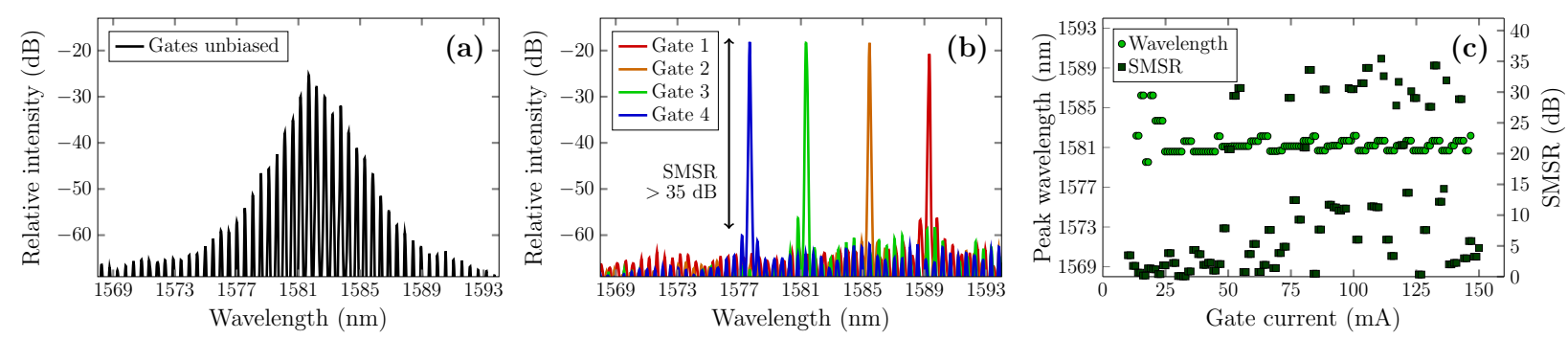

(d)
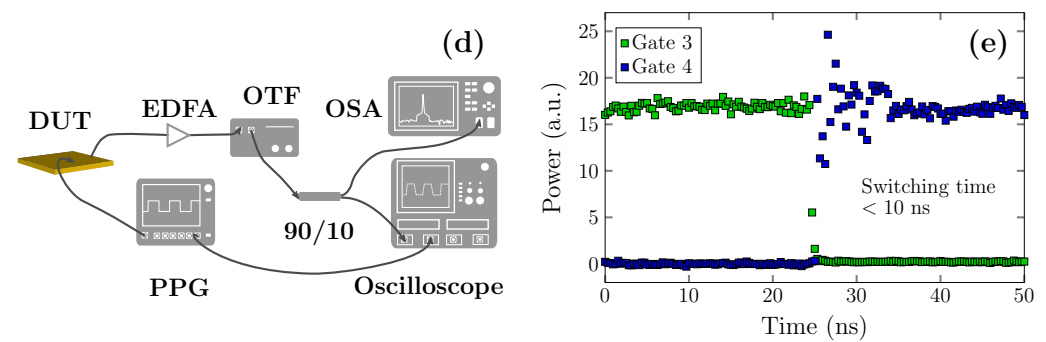

Channel $1-12.5 \mathrm{Gbit} / \mathrm{s}$

(f)

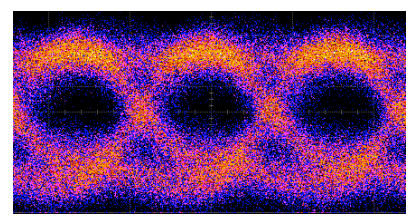

Fig. 2. (a) Optical spectrum at a gain current of $100 \mathrm{~mA}$ with unbiased gates. (b) Lasing spectra at a gain current of $100 \mathrm{~mA}$ with the different gates biased above transparancy. (c) Wavelength and SMSR dependence on the gate current (Gate 3). (d) Optical setup to measure the wavelength switching speed. (e) Wavelength switching behavior between Gate 3 and Gate 4. (f) Eye diagram for direct modulation at $12.5 \mathrm{Gbit} / \mathrm{s}$ (Gate 1).

\section{Conclusion}

We have demonstrated fast wavelength switching with a III-V-on-silicon laser that exploits filtered feedback to achieve single-mode lasing. Switching times for stable operation are less than $10 \mathrm{~ns}$ and each wavelength channel can be directly modulated at $12.5 \mathrm{Gbit} / \mathrm{s}$, making the structure very attractive for use in optical packet or burst switching systems.

\section{References}

1. M. A. Mestre, et al., J. Lightw. Technol. 34(8), 1851-1858 (2016).

2. S. Dhoore, et al., Opt. Lett. 42(6), 1121-1124 (2017).

3. B. Docter, et al., IEEE J. Sel. Topics Quantum Electron. 16(5), 1405-1412 (2010).

4. G. Roelkens, et al., Photonics 2(3), 969-1004 (2015). 